\title{
A Survey on Ethnoveterinary Medicines used by the Tribal Peoples of Kalasapadi Hills, Dharmapuri District of Tamil Nadu
}

\author{
${ }^{1}$ Rajesh P, ${ }^{2}$ Meenakshi R, ${ }^{3}$ Rajkumar R, ${ }^{4}$ Selvam K \\ Department of Botany, School of Life Sciences, Periyar University \\ Salem, Tamil Nadu, India
}

\begin{abstract}
Objective: This study aims at providing a comprehensive documentation on ethanoveterinary plant knowledge of the tribal people in order to preserve the fost-eroding knowledge and resources of the kalasapadi hills, Pappireddipatti revenue Tk, Dharmapuri.
\end{abstract}

Methods: Field work was conducted from 20152016. Moreover 36 informants were interviewed. First, successive oral free listing and semi-structured interviews were performed. The veterinary diseases as described by the informants were categorized according to the symptoms they cause and the organs they affect. Information on the cited plants, informant consensus factor (ICF) and fidelity level (FL) was calculated based on use reports.

Results: Utilization of 49 plant species, belonging to 30 genera under 31 families, has been recorded against livestock ailments. Plant parts, such as leaf, root, flower, bark, resin, and rhizome, are used in the preparation. Among the plant parts, bark is predominately used. Most of the preparations include parts of more than one plant as the ingredients, and many of such combined preparations are used for treating more than one ailment.

Conclusion: According to the local people, the most often mentioned species have high medicinal potential. At the same time the comprehensive pharmacological investigations of the herbal plants will be helpful in development of new drugs for a particular condition. There is a need to conserve the

knowledge of medicinal plant use in the Kalasapadi hills for future generations and to incorporate it into existing livestock health care services.

Keywords: Ethno-veterinary medicine, Malayali tribes, Livestock, Kalasapadi, Kalrayan hills.

\section{Introduction:}

India is rich in plant diversity and possesses almost $7 \%$ of the world's flowering plants. The Eastern Ghats of India are endowed with an extensively rich variety of biological species, geographical formations, and diverse ethnic tribes. Ethnomedicinal studies in the Eastern Ghats of Tamil Nadu have been carried out previously by a number of researchers $[1,2]$. There is abundant undocumented traditional knowledge of herbal remedies used to treat animal diseases in most cultures [3]. Indian therapeutic system adopted observations and rational procedures by the end of the Vedic eara. This led to the foundations of a new medicinal system known as Ayurveda. The knowledge formed the basis for veterinary medicine [4]. In India, ancient literature such as the Vedas, and other written scripuatures like Scand Puran (2350 BC), Cherak and shusrutha (2500-600 BC), have long documented the treatment of animal disease by using medicinal plants $[5,6]$ The uses of a protective ointment for human beings, cows and horses have been documented in the Atharva Veda (IV, 9, 2) [7]. Traditional ethnoveterinary medicine (EVM) prepared by humans, for the purposes of maintaining or 
restoring animal health, plays a significant role in several regions of the world especially in areas where cattle is a major source of income for rural peoples [8]. In certain areas, these folk medical prescriptions are endemic and have survived throughout ages from one generation to the next throughout spiritual word of mouth. They do not exist as written knowledge. Indigenous people developed this knowledge through practical experience and skill to solve the problems that they faced on day to day activity $[9,10]$. The treatment of animal diseases often parallels the treatment of human illness, as do the fundamental concepts [11] and the similar therapeutic plants may be used for both, humans and animals [12]. Especially in mountainous and high elevation areas, cattle is very important for local economies, food and social security, and is often a symbol of prestige [13, 14]. Livelihood strategies of rural people heavily depend on livestock farming, particularly in developing countries [15]. Livestock plays a vital role in (Indian) farmer's life. It gives manure, fuel, milk and meat, etc and also generates rural economy and rural employment. Farmers take care of their livestock using EVM. These medicines are cheaper than western drug [16, 17]. According to [18], we also determined the frequency index (FI), informant consensus factor (ICF) of the ethno-veterinary diseases and use-value of the plant species. Cattle are considered as the wealth of the tribal people, and their well-being is considered to be very important [19]. As the tribal people are enriched with traditional ethnoveterinary knowledge, the forest resources in their surroundings play a very significant role in their routine life [20], and medicinal plants are the chief source for the primary health-care services of such rural population [21]. Ethno-veterinary medicine (EVM) is defined as the traditional animal healthcare which comprises the folk knowledge, believes, practices, skills, methods, and practices pertaining to health care of animals [22, 23]. As the villagers around the globe are having limited access to modern medicine, they strictly depend on the traditional medicine for the health care of livestock. Regrettably, these practices are not properly documented and largely lost because they are passed on generation through verbal communication and dilution due to lack of observation and technical skills. EVM contributes in the management of animal diseases in a cost-effective manner but unfortunately research works, which prove the pharmaceutical efficiency of plants, have not been done so far, or a very little research work has been done [24]. It is an established fact that plants serve as potent medicines for curing various diseases of the tribal community as well as their livestock [25]. As $80 \%$ of populations from the developed countries are depend on traditional medicine prepared from the medicinal plants, it is necessary to investigate them for understanding their properties, safety, and their efficacy [26]. Keeping this in view, the present study was initiated to explore plants utilized for ethnoveterinary health care and document the traditional veterinary medicinal knowledge of Kalasapadi hills Malayali tribes.

\section{MATERIALS AND METHODS}

\subsection{Study area}

The present study area was conducted among the Kalasapadi hamlets, west part of the Kalrayan hills in Pappireddipatti block of Dharmapuri district, Tamil Nadu, India (Fig. 1). The total extends of Pappireddipatti taluk (Sub-district) is $788.3 \mathrm{Sq} . \mathrm{km}$ (Statistical handbook-2007-08). Kalasapadi is located at $11^{\circ} 54^{\prime}$ and $4866^{\prime \prime} \mathrm{N}$ latitude and $78^{\circ} 29^{\prime}$ and $1236^{\prime \prime}$ E longitudes and altitude varies from 252 to $3783 \mathrm{ft}$ (etrex 20-Germin) the highest peak (Bootu malai) of the hills is 1153 meters above mean sea level. The average annual rainfall ranges from 783.97 to 1582.20 $\mathrm{mm}$, and the temperature varies from $25^{\circ} \mathrm{C}$ to $35^{\circ} \mathrm{C}$. Vegetation of Kalrayan hills is semi-deciduous to scrub forests where sandal wood grows naturally along with other dominant species like teak and bamboo. Indigenous species like Terminalia chebula, Pterocarpus marsupium and Dalbergia latifolia also found abundant in this region [27].

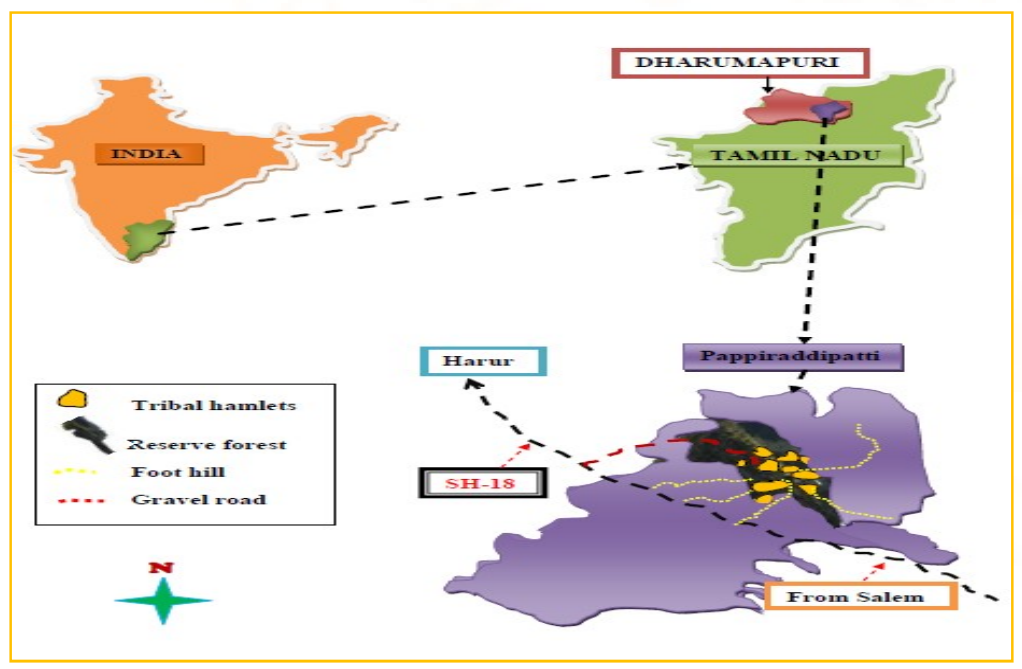

Fig 1: Location map of study area 


\subsection{Tribal community}

Native tribal people of Karayan hills are called Malayali (lit. Malai = hill; alu = inhabit) [28], their language has been formed by the place where they stayed [1]. The native people of the Kalrayans were called as Vedar (Hunter). The warriors belonging to Karalar community had invaded from Kanchipuram district and settled in Kalrayan hills. The mixed population of Karalar and Vedar communities presently called Malayali and they call themselves as malayala goundars [29]. Some of them are farmers and remaining People depend on works interrelated with agriculture and involved in livestock keeping, poultries, collection of honey, bee wax, and other minor forest products [30].

\subsection{Data Collection}

A systematic and extensive ethno veterinary medicine survey was carried out in Kalasapadi hills during Jan 2015 to March 2016 following the standard protocols for the collection of ethno- botanical data [31, 32]. Ethno veterinary information and EVM importance of plants were collected from traditional healers, aged persons, and farmers having familiarity and knowledge with plants by following standard methods such as personal interviews, discussions, and field observations through regular field visits to various hamlets. The information on EVM was cross verified by the communication with traditional healers and other knowledgeable persons, elders, farmers, livestock owners, informants residing in nearby area. The plants of EVM interest were identified and verified with standard flora available $[33,34]$ and the voucher specimens were deposited in Department of Botany, Periyar University, Salem. EVM plants enumerated according to the ailments cured in alphabetical sequence of the botanical name in each ailment with vernacular name followed by family, plant part used, mode of preparation, administration, and dosage required.

\subsection{Data analysis}

The informant or respondent consensus factor (FIC) (Table. 3) was calculated to estimate user variability of medicinal plants $[35,36]$. FIC values range from 0.00 to 1.00 . High FIC values are obtained when only one or a few plant species are reported to be used by a high proportion of informants to treat a particular ailment, whereas low FIC values indicate that informants disagree over which plant to use [35].
High FIC values can thus be used to pinpoint particularly interesting species for the search of bioactive compounds [36]. FIC is calculated using the following formula [37].Most frequently used plants for different ailment categories based on maximum FL $(\%)$ in each ailment category

$$
\mathrm{FL}=\frac{N p}{N \times 100}
$$

Where $\mathrm{Np}=$ number of informants that claim a use of a plant species to treat a particular disease; $\mathrm{N}=$ number of informants that use the plants as a medicine to treat any given disease [31]. Informant consensus factor the informant consensus factor (FIC) was used to see if there was agreement in the use of plants in the ailment categories between the plant users in the study area. The Fic was calculated using the following formula [35]. Where Nur refers to the number of use-reports for a particular ailment category and $\mathrm{Nt}$ refers to the number of taxa used for aparticular ailment category by all informants. The product of this factor ranges from 0 to 1 . A high value (close to 1.0) indicates that relatively few taxa are used by a large proportion of the informants. A low value indicates that the informants disagree on the taxa tobe used in the treatment within a category of illness. Where Nur is the number of individual plant use reports for a particular illness category, and $\mathrm{Nt}$ is the total number of species used by all informants for this illness category. The fidelity level (FL) The fidelity level (FL), the percentage of informants claiming the use of a certain plant for the same major purpose, was calculated for the most frequently reported and men, and among respondents[38]. The women learn from their mothers or fathers through routine observations while their fathers' teach the man, in addition to routine observations, since the traditional knowledge in the family or community is passed from male parent to his first-born son [39]. Categories of ailments and informant consensus factor (FIC) for each category

Where, Nur $=$ number of use reports from informants for a particular plant-use category; $\mathrm{Nt}=$ number of taxa or species that are used for that plant use category for all informants.

$$
\mathrm{F}_{\mathrm{ic}}=\frac{N u r-N t}{N u r-1}
$$




\section{RESULTS}

The traditional knowledge of tribal communities of Kalasapadi, west part of the Kalrayan hills, Eastern Ghats of India had high ethno veterinary importance. People utilized numerous plants and their various parts. Apart from the various plant parts, healers use certain animal products as the ingredients in the medicaments such as pig ghee, egg white yolk, cow dung, cow milk, goat milk, buttermilk, animal flesh, and children's urine. Describes the plants used by its diseases followed by botanical name which it belongs to family name, traditional name, life form, parts use, mode of preparation, other ingredients, route of administration, dosage and duration of treatment methods of preparation and dosage by farmers, and validity scores as per prepared questionnaire. The present study revealed 49 plant species belonging to 30 genera and 31 families commonly (Table.1).

The plant species used are commonly available in the region and play special role in veterinary medicine. Various animal conditions were identified in the study area. The present study highlights the EVM knowledge and practices found among the tribes of Kalasapadi hills of the study area. Through the discussion with the native people, we understand the population of cattle has been reduced in the study area. The main factor for this decrease is the profligate returns due to the low productivity and replacement of livestock in the agricultural practices by the machinery. Most of the tribes are aware of the traditional EVM system, but all of them are not practically using them. During the study, we came across the traditional knowledge of Malayali tribes of Kalasapadi hills on EVM.

\subsection{Habit and habitat of medicinal plants}

Nearly all of the medicinal plants (Fig. 2) were collected from the wild $(89 \%)$ and others were from dwelling gardens(11\%), the habit of the medicinal plants indicated that most of them trees and shrubs dominate with $(57.14 \%)$,species followed by herbs $(26.53 \%)$, climbers $(14.28 \%)$, and Prostrate herb $(2.04 \%)$.

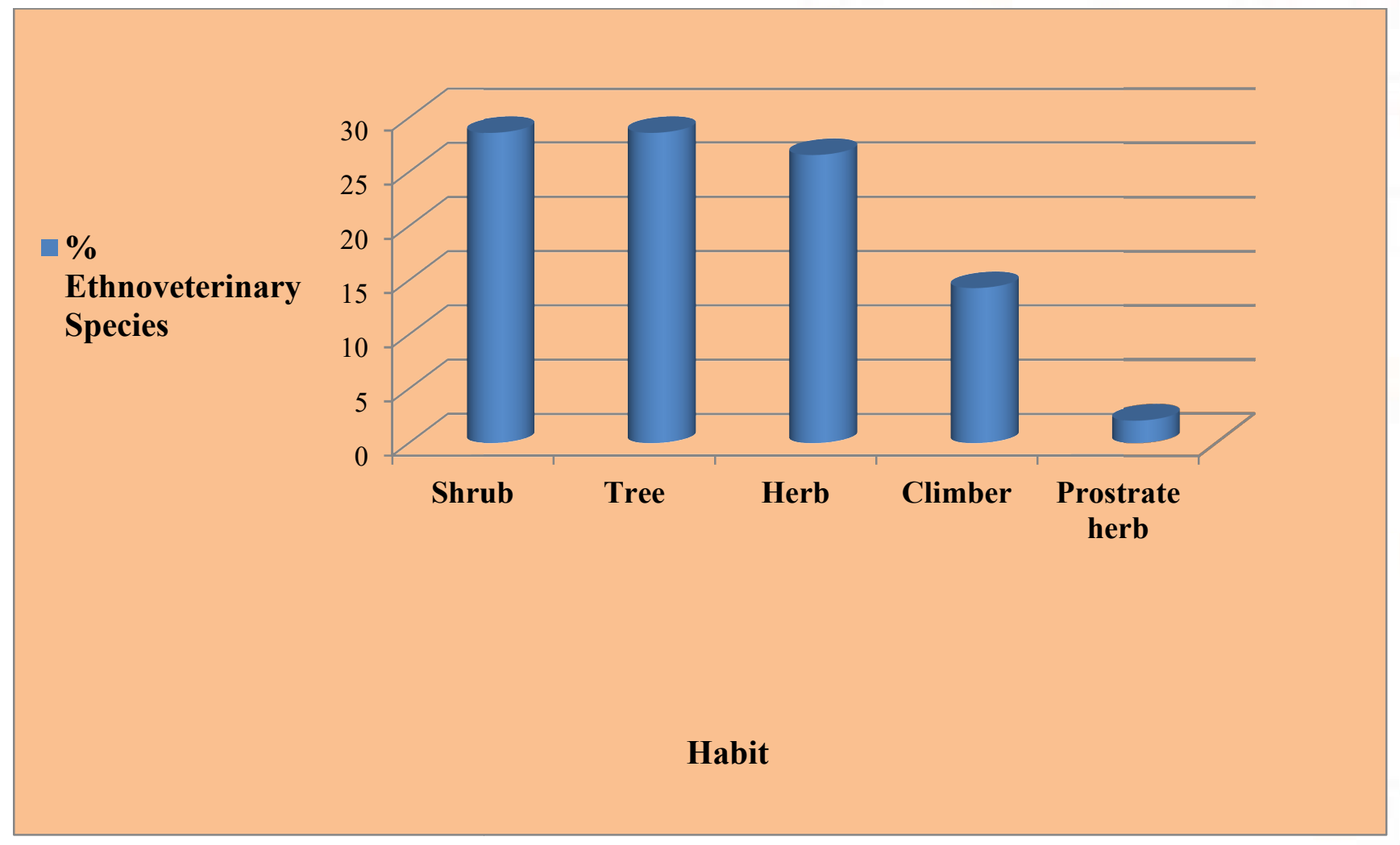

Fig 2: Habit 
Table 1: List of Disease and medicinal plants used by local Malayali Tribales in Kalasapadi hills

\begin{tabular}{|c|c|c|c|c|c|c|c|c|}
\hline Diseases & $\begin{array}{l}\text { Botanical } \\
\text { name }\end{array}$ & Family & $\begin{array}{l}\text { Traditiona } \\
\text { I name }\end{array}$ & $\begin{array}{l}\text { Life } \\
\text { form }\end{array}$ & $\begin{array}{l}\text { parts } \\
\text { use }\end{array}$ & $\begin{array}{l}\text { Mode } \\
\text { of } \\
\text { Prepa } \\
\text { ration }\end{array}$ & $\begin{array}{l}\text { Other } \\
\text { ingredi } \\
\text { ents }\end{array}$ & $\begin{array}{c}\text { Route of } \\
\text { administratio } \\
\text { n, dosage and } \\
\text { duration of } \\
\text { treatment }\end{array}$ \\
\hline $\begin{array}{l}\text { Dysenter } \\
\text { y with } \\
\text { blood } \\
\text { (Ratha } \\
\text { kalichal) }\end{array}$ & $\begin{array}{l}\text { Carmona retusa } \\
\text { Cassia } \\
\text { auriculata } \\
\text { Flueggea } \\
\text { leucopyrus }\end{array}$ & $\begin{array}{l}\text { Boraginaceae } \\
\text { Fabaceae } \\
\text { phyllanthacea } \\
\text { e }\end{array}$ & $\begin{array}{l}\text { Kurangu } \\
\text { vethalai } \\
\text { Avaram } \\
\text { Vara poola }\end{array}$ & $\begin{array}{l}\text { Shrub } \\
\text { Shrub } \\
\text { Shrub }\end{array}$ & $\begin{array}{l}\text { Leaf } \\
\text { Leaf } \\
\text { Leaf }\end{array}$ & $\begin{array}{l}\text { Paste } \\
\text { Paste } \\
\text { Paste }\end{array}$ & $\begin{array}{l}\text { butter } \\
\text { milk } \\
\text { butter } \\
\text { milk } \\
\text { butter } \\
\text { milk }\end{array}$ & $\begin{array}{l}3 \text { tender leaves } \\
\text { of each plant } \\
\text { are ground } \\
\text { together and } \\
\text { paste into a } \\
\text { Lemon juice } \\
\text { and add to a } \\
\text { butter milk is } \\
\text { given orally } \\
\text { twice a day for } \\
3 \text { or } 4 \text { days or } \\
\text { up to cure. }\end{array}$ \\
\hline $\begin{array}{l}\text { Mastitis } \\
\text { (blood in } \\
\text { milk ) }\end{array}$ & $\begin{array}{l}\text { Asparagus } \\
\text { rescemosus }\end{array}$ & $\begin{array}{l}\text { Lillieaceae } \\
\text { Convolvulace } \\
\text { ae } \\
\text { Asteraceae } \\
\text { Zingiberacea } \\
\text { e }\end{array}$ & $\begin{array}{l}\text { Thanner } \\
\text { vettan } \\
\text { khilangu } \\
\text { Ushnaganthi }\end{array}$ & $\begin{array}{l}\text { climber } \\
\text { Herb } \\
\text { shrub } \\
\text { Herb }\end{array}$ & $\begin{array}{l}\text { Root } \\
\text { tuber }\end{array}$ & Paste & - & $\begin{array}{l}\text { Root and leaf } \\
\text { crused, in paste } \\
\text { from these are } \\
\text { externally } \\
\text { applied on the } \\
\text { infected area. } \\
\text { Leaves and } \\
\text { Curcuma longa } \\
\text { (Fresh } \\
\text { rhizome) are } \\
\text { crused, mixed } \\
\text { with Ricinus } \\
\text { communis oil } \\
\text { and are } \\
\text { externally used } \\
\text { to cure } \\
\text { Mastitis. }\end{array}$ \\
\hline $\begin{array}{l}\text { Fever } \\
\text { (kunthu) }\end{array}$ & $\begin{array}{l}\text { Cadaba } \\
\text { fruticosa } \\
\text { (L.)Druce } \\
\text { Toddalia } \\
\text { asiatica } \\
\text { (L.)Lam } \\
\text { Calotropis } \\
\text { gigantea (L.) } \\
\text { W.T.Aiton }\end{array}$ & $\begin{array}{l}\text { Capparaceae } \\
\text { Rutaceae } \\
\text { Asclepiadac } \\
\text { eae }\end{array}$ & $\begin{array}{l}\text { Mullu } \\
\text { ellamolagar } \\
\text { anai } \\
\text { Mullu } \\
\text { molagaranai } \\
\text { Earukku }\end{array}$ & $\begin{array}{l}\text { shrub } \\
\text { Shrub }\end{array}$ & $\begin{array}{l}\text { Leaf } \\
\text { Leaf }\end{array}$ & Paste & $\begin{array}{l}\text { Hot } \\
\text { water } \\
\text { Hot } \\
\text { water } \\
\text { Hot } \\
\text { water }\end{array}$ & $\begin{array}{l}3 \text { tender } \\
\text { leaves of each } \\
\text { plant are } \\
\text { ground } \\
\text { together and } \\
\text { paste and add } \\
5 \text { pepper } \\
\text { (milagu) and } \\
\text { (vellaipoondu } \\
\text { )Allium ativam at } \\
\text { sativa }\end{array}$ \\
\hline
\end{tabular}




\begin{tabular}{|c|c|c|c|c|c|c|c|c|}
\hline & & & & & & & & $\begin{array}{l}\text { bearable heat } \\
\text { decoction is } \\
\text { administered } \\
\text { orally two or } \\
\text { three tumbler } \\
\text { for } 1 \text { or } 2 \text { days } \\
\text { in the } \\
\text { morning and } \\
\text { evening. }\end{array}$ \\
\hline $\begin{array}{l}\text { Parturit } \\
\text { ion } \\
\text { (Delivery } \\
\text { problems } \\
\text { and } \\
\text { release } \\
\text { of } \\
\text { retained } \\
\text { placenta) }\end{array}$ & $\begin{array}{l}\text { Grewia asiatica } \\
\text { Bauhnia } \\
\text { recemosa }\end{array}$ & $\begin{array}{l}\text { Tiliaceae } \\
\text { Fabaceae }\end{array}$ & $\begin{array}{l}\text { Thadasa } \\
\text { maram } \\
\text { Sitathi }\end{array}$ & $\begin{array}{l}\text { Tree } \\
\text { Tree }\end{array}$ & $\begin{array}{l}\text { Leaf } \\
\text { Leaf }\end{array}$ & $\begin{array}{l}\text { Raw } \\
\text { Raw }\end{array}$ & - & $\begin{array}{l}20-25 \text { leaves } \\
\text { are given the } \\
\text { feed orally at } \\
\text { the time of } \\
\text { delivery which } \\
\text { regulates the } \\
\text { proper } \\
\text { elimination of } \\
\text { placenta. }\end{array}$ \\
\hline Dog bite & $\begin{array}{l}\text { Achyranthes } \\
\text { aspera Linn. } \\
\text { Calotropis } \\
\text { procera A. }\end{array}$ & $\begin{array}{l}\text { Amaranthace } \\
\text { ae } \\
\text { Asclepiadace } \\
\text { ae }\end{array}$ & Nayuruvi & Herb & $\begin{array}{l}\text { Whole } \\
\text { plant }\end{array}$ & Juice & $\begin{array}{l}\text { Slaked } \\
\text { lime }\end{array}$ & $\begin{array}{l}\text { Slaked limes } \\
\text { with Nayuruvi } \\
\text { leaf like juice } \\
\text { are exteranally } \\
\text { applied on the } \\
\text { infected area. } \\
\text { Tender twig } \\
\text { latex applies } \\
\text { for externally. }\end{array}$ \\
\hline \multirow[t]{2}{*}{$\begin{array}{l}\text { Eye } \\
\text { wounds } \\
\text { due to } \\
\text { stick } \\
\text { injuries } \\
\text { (kuchi } \\
\text { kannil } \\
\text { kuthutha } \\
\text { l) }\end{array}$} & $\begin{array}{l}\text { Borassus } \\
\text { flabellifer } \\
\text { L. }\end{array}$ & $\begin{array}{l}\text { Euphorbiacea } \\
\text { e }\end{array}$ & $\begin{array}{l}\text { Muthu } \\
\text { kottai }\end{array}$ & Tree & $\begin{array}{l}\text { Leaf } \\
\text { petiole }\end{array}$ & Juice & Salt & $\begin{array}{l}\text { Borassus } \\
\text { flabellifer leaf } \\
\text { petiole and } \\
\text { heating are } \\
\text { taken in equal } \\
\text { amount of salt } \\
\text { pound and } \\
\text { juice is applied } \\
\text { externally as } \\
\text { drops in eyes } \\
\text { through white } \\
\text { cloth } 2 \text { or } 3 \\
\text { times up to } \\
\text { cure. } \\
\text { Oil is applied } \\
\text { externally as } \\
\text { drops in eyes } \\
\text { through finger2 } \\
\text { or } 3 \text { times up to } \\
\text { cure. }\end{array}$ \\
\hline & $\begin{array}{l}\text { Decalepis } \\
\text { hamiltonii }\end{array}$ & $\begin{array}{l}\text { Dioscoreacea } \\
\text { e }\end{array}$ & $\begin{array}{l}\text { Peru } \\
\text { maviligam }\end{array}$ & Climber & Root & Paste & - & Fresh root \\
\hline
\end{tabular}




\begin{tabular}{|c|c|c|c|c|c|c|c|c|}
\hline $\begin{array}{l}\text { Lactatio } \\
\text { n } \\
\text { (Milk } \\
\text { yielding) }\end{array}$ & $\begin{array}{l}\text { wight\&Arm } \\
\text { Henidesmus } \\
\text { indicus(L).R.Br } \\
\text {, }\end{array}$ & $\begin{array}{l}\text { Asclepiadace } \\
\text { ae }\end{array}$ & $\begin{array}{l}\text { Seru } \\
\text { maviligam }\end{array}$ & Climber & Root & Paste & - & $\begin{array}{l}\text { grain Fresh } \\
\text { root used as } \\
\text { fodder } \\
\text { increase } \\
\text { lactation. } \\
\text { Fresh root } \\
\text { grain Fresh } \\
\text { root used as } \\
\text { fodder } \\
\text { increase } \\
\text { lactation. }\end{array}$ \\
\hline $\begin{array}{l}\text { Feeding } \\
\text { problem } \\
\text { (Theevan } \\
\text { am } \\
\text { utkollam } \\
\text { ai) }\end{array}$ & $\begin{array}{l}\text { Cissus } \\
\text { quadrangularis } \\
\text { L. } \\
\text { Caralluma } \\
\text { umbellata } \\
\text { Haw. }\end{array}$ & $\begin{array}{l}\text { Vitaceae } \\
\text { Asclepiadac } \\
\text { eae }\end{array}$ & $\begin{array}{l}\text { Perandai } \\
\text { Kallu } \\
\text { Mulaiyan }\end{array}$ & $\begin{array}{l}\text { Climber } \\
\text { Herb }\end{array}$ & $\begin{array}{l}\text { Stem } \\
\text { Stem }\end{array}$ & $\begin{array}{l}\text { Paste } \\
\text { Paste }\end{array}$ & Salt & $\begin{array}{l}\text { Stem half } \\
\text { paste and } \\
\text { ordinary salt } \\
\text { is applied in } \\
\text { tongue } \\
\text { externally. }\end{array}$ \\
\hline $\begin{array}{l}\text { Snake } \\
\text { bite }\end{array}$ & $\begin{array}{l}\text { Andrographis } \\
\text { lineate Wall. } \\
\text { Andrographis } \\
\text { paniculata(Bur } \\
\text { m.F.) } \\
\text { Aristolochia } \\
\text { bracteolate } \\
\text { Lam. }\end{array}$ & $\begin{array}{l}\text { Acanthaceae } \\
\text { Aristolochiac } \\
\text { eae }\end{array}$ & $\begin{array}{l}\text { Periyananga } \\
\text { i } \\
\text { Siriyanangai } \\
\text { Aaduthinda } \\
\text { paalai }\end{array}$ & $\begin{array}{l}\text { Herb } \\
\text { Herb }\end{array}$ & $\begin{array}{l}\text { Leaf } \\
\text { Leaf }\end{array}$ & $\begin{array}{l}\text { Decoct } \\
\text { ion } \\
\text { Decoct } \\
\text { ion }\end{array}$ & $\begin{array}{l}\text { Hot } \\
\text { water } \\
\text { Hot } \\
\text { water } \\
\text { Hot } \\
\text { water }\end{array}$ & $\begin{array}{l}\text { Each three } \\
\text { plant dry power } \\
\text { with Hot water } \\
\text { and } \\
\text { administered } \\
\text { orally } \\
\text { (sooranum) and } \\
4 \text { or } 5 \text { pepper } \\
\text { are taken } \\
\text { internally cure } \\
\text { snake bite. }\end{array}$ \\
\hline $\begin{array}{l}\text { Sprain(s } \\
\text { ulukku } \\
\text { pidithal) }\end{array}$ & $\begin{array}{l}\text { Ricinus } \\
\text { communis L } \\
\text { Eleasine } \\
\text { coracana } \\
\text { Gaertn. }\end{array}$ & $\begin{array}{l}\text { Euphorbiacea } \\
\mathrm{e} \\
\text { Poaceae }\end{array}$ & $\begin{array}{l}\text { Muthu } \\
\text { kottai } \\
\text { Aarium(kev } \\
\text { uru ) }\end{array}$ & $\begin{array}{l}\text { Shrub } \\
\text { Herb }\end{array}$ & $\begin{array}{l}\text { seed } \\
\text { Grains }\end{array}$ & $\begin{array}{l}\text { oil } \\
\text { powder }\end{array}$ & $\begin{array}{l}- \\
\text { Hot } \\
\text { water }\end{array}$ & $\begin{array}{l}\text { Oil is applied } \\
\text { externally as } \\
\text { drops in eyes } \\
\text { through finger } 2 \\
\text { or } 3 \text { times up to } \\
\text { cure } \\
\text { Make into a } \\
\text { paste apply on } \\
\text { the skin for } \\
\text { Sprain. }\end{array}$ \\
\hline $\begin{array}{l}\text { Wound } \\
\text { (Thorns } \\
\text { in legs ) }\end{array}$ & $\begin{array}{l}\text { Calotropis } \\
\text { gigantea (L.) } \\
\text { W.T.Aiton } \\
\text { Cleistanthus } \\
\text { collinus } \\
\text { (Roxb.) }\end{array}$ & $\begin{array}{l}\text { Asclepiadac } \\
\text { eae }\end{array}$ & $\begin{array}{l}\text { Oduvanthal } \\
\text { ai }\end{array}$ & Shrub & Latex & Paste & - & $\begin{array}{l}\text { Tender twig } \\
\text { latex apply for } \\
\text { externally } \\
\text { Leaf paste } \\
\begin{array}{l}\text { applying for } \\
\text { externally }\end{array}\end{array}$ \\
\hline
\end{tabular}




\begin{tabular}{|c|c|c|c|c|c|c|c|c|}
\hline & Hook.f. & & & & & & & wounded area. \\
\hline $\begin{array}{l}\text { Wounds } \\
\text { and } \\
\text { injuries }\end{array}$ & $\begin{array}{l}\text { Evolvulus } \\
\text { alsinoides } \\
\text { (Linn.) } \\
\text { Lantana } \\
\text { camera.L }\end{array}$ & $\begin{array}{l}\text { Convolvulace } \\
\text { ae } \\
\text { Verbenacea } \\
\text { e }\end{array}$ & $\begin{array}{l}\text { Uishnaganth } \\
\text { i } \\
\text { Uni mul }\end{array}$ & $\begin{array}{l}\text { climber } \\
\text { shrub }\end{array}$ & $\begin{array}{l}\text { Leaf } \\
\text { Leaf }\end{array}$ & $\begin{array}{l}\text { paste } \\
\text { paste }\end{array}$ & $\begin{array}{l}\text { Slaked } \\
\text { lime } \\
\text { Slaked } \\
\text { lime }\end{array}$ & $\begin{array}{l}\text { Leaf paste and } \\
\text { (slaked lime) } \\
\text { applied } \\
\text { on the Wound } \\
\text { part externally. }\end{array}$ \\
\hline $\begin{array}{l}\text { Yoke gal } \\
\text { (kaluthu } \\
\text { katti) }\end{array}$ & $\begin{array}{l}\text { Coccinia indica } \\
\text { Wight\&Arn. } \\
\text { Perularia } \\
\text { extensa } \\
\text { (jacq.)N.E.Br }\end{array}$ & $\begin{array}{l}\text { Cucurbitacea } \\
\text { e } \\
\text { Asclepiadace } \\
\text { ae }\end{array}$ & $\begin{array}{l}\text { Kovai kodi } \\
\text { Veliparuthi }\end{array}$ & $\begin{array}{l}\text { Climber } \\
\text { climber }\end{array}$ & $\begin{array}{l}\text { Leaf } \\
\text { Leaf }\end{array}$ & $\begin{array}{l}\text { Juice } \\
\text { Juice }\end{array}$ & $\begin{array}{l}\text { Salt } \\
\text { Salt }\end{array}$ & $\begin{array}{l}\text { Leaf juice and } \\
\text { salt applied } \\
\text { On externally. }\end{array}$ \\
\hline $\begin{array}{l}\text { Cancer } \\
\text { (puthu } \\
\text { kattigal) }\end{array}$ & $\begin{array}{l}\text { Endostemon } \\
\text { viscosus } \\
\text { (Roth)M.R.Ash } \\
\text { by } \\
\text { Hardwickia } \\
\text { binata Roxb }\end{array}$ & $\begin{array}{l}\text { Lamiaceae } \\
\text { Fabaceae }\end{array}$ & $\begin{array}{l}\text { Kaayapathin } \\
\text { i } \\
\text { Aachaa }\end{array}$ & $\begin{array}{l}\text { Herb } \\
\text { Tree }\end{array}$ & $\begin{array}{l}\text { Leaf } \\
\text { Root } \\
\text { Bark }\end{array}$ & paste & - & $\begin{array}{l}\text { Leaf grind } \\
\text { well into paste } \\
\text { mixed with } \\
\text { salt and apply } \\
\text { externally } \\
\text { Root bark } \\
\text { exudates are } \\
\text { applied } \\
\text { externally. }\end{array}$ \\
\hline $\begin{array}{l}\text { Animal } \\
\text { uniformt } \\
\text { y }\end{array}$ & $\begin{array}{l}\text { Begonia Sp., } \\
\text { Peperomia } \\
\text { blanda (Jacq.) }\end{array}$ & $\begin{array}{l}\text { Begonieaceae } \\
\text { Piperaceae }\end{array}$ & $\begin{array}{l}\text { Orukai } \\
\text { thalai } \\
\text { Sangalapoo } \\
\text { ndu }\end{array}$ & $\begin{array}{l}\text { Herb } \\
\text { Herb }\end{array}$ & $\begin{array}{l}\text { Leaf } \\
\text { Leaf }\end{array}$ & $\begin{array}{l}\text { Juice } \\
\text { juice }\end{array}$ & $\begin{array}{l}\text { Water } \\
\text { Water }\end{array}$ & $\begin{array}{l}\text { Cattles are } \\
\text { always in } \\
\text { groups the won } \\
\text { disturbed other } \\
\text { animals. }\end{array}$ \\
\hline $\begin{array}{l}\text { Mesmeri } \\
\text { sm } \\
\text { (vasiyam } \\
\text { ) }\end{array}$ & $\begin{array}{l}\text { Mimosa } \\
\text { pudica L. } \\
\text { Drosera } \\
\text { burmannii vahl }\end{array}$ & $\begin{array}{l}\text { Mimosaceae } \\
\text { Droseraceae }\end{array}$ & $\begin{array}{l}\text { Thotta } \\
\text { Sinungi } \\
\text { Alukanni }\end{array}$ & $\begin{array}{l}\text { Herb } \\
\text { Prostrate } \\
\text { herb }\end{array}$ & $\begin{array}{l}\text { Leaf } \\
\text { Whole } \\
\text { plant }\end{array}$ & $\begin{array}{l}\text { Paste } \\
\text { Paste }\end{array}$ & - & $\begin{array}{lr}\text { Hole } & \text { plant } \\
\text { crused } & \text { and } \\
\text { mixed } & \text { the } \\
\text { coconut } & \text { oil } \\
\text { apply } & \text { the } \\
\text { animal. } & \end{array}$ \\
\hline $\begin{array}{l}\text { Bone } \\
\text { fracture } \\
\text { (Ealumb } \\
\text { u } \\
\text { murivu) }\end{array}$ & $\begin{array}{l}\text { Albizia } \\
\text { procera } \\
\text { Benth. } \\
\text { Artocarpus } \\
\text { hirsutus Lam. }\end{array}$ & Fabaceae & $\begin{array}{l}\text { Kudumada } \\
\text { duri }\end{array}$ & $\begin{array}{l}\text { Tree } \\
\text { Tree }\end{array}$ & Bark & $\begin{array}{l}\text { Paste } \\
\text { Paste }\end{array}$ & $\begin{array}{l}\text { Egg(naa } \\
\text { tukholi) }\end{array}$ & $\begin{array}{l}\text { Albizia } \\
\text { procera or } \\
\text { Artocarpus } \\
\text { hirsutus bark } \\
\text { are grind well } \\
\text { with egg } \\
\text { white yolk } \\
\text { and made into } \\
\text { solid paste } \\
\text { Paste } \\
\text { applied is } \\
\text { externally on } \\
\text { the area up to } \\
\text { cure. }\end{array}$ \\
\hline
\end{tabular}




\begin{tabular}{|c|c|c|c|c|c|c|c|c|}
\hline $\begin{array}{l}\text { Sokku } \\
\text { (Spiritua } \\
\text { l) }\end{array}$ & $\begin{array}{l}\text { Calotropis } \\
\text { gigantea } \\
\text { Tamarindus } \\
\text { indica } \mathrm{L}\end{array}$ & $\begin{array}{l}\text { Asclepiadac } \\
\text { eae } \\
\text { Caesalpinia } \\
\text { ceae }\end{array}$ & Erukku & Shrub & Fruit & Paste & Water & $\begin{array}{l}\text { We have to } \\
\text { collect soil } \\
\text { near the roots } \\
\text { of Calotropis } \\
\text { gigantea and } \\
\text { round that } \\
\text { plant three } \\
\text { times. } \\
\text { Fruit are used } \\
\text { in iternal } 500 \\
\text { ml. Spiritual. }\end{array}$ \\
\hline $\begin{array}{l}\text { Komari } \\
\text { (foot } \\
\text { and } \\
\text { mouth } \\
\text { disease) }\end{array}$ & $\begin{array}{l}\text { Musa } \\
\text { paradisiaca } \\
\text { L. } \\
\\
\text { Limo } \\
\text { nia } \\
\text { acidis } \\
\text { sima } \\
\text { L }\end{array}$ & $\begin{array}{l}\text { Musaceae } \\
\text { Rutaceae }\end{array}$ & $\begin{array}{l}\text { Monthan } \\
\text { Valai } \\
\text { Vila }\end{array}$ & Tree & Fruit & - & $\begin{array}{l}\text { pig ghee } \\
\text { pig ghee }\end{array}$ & $\begin{array}{l}\text { Two or three } \\
\text { fruits are } \\
\text { longitudinally } \\
\text { spilit into two } \\
\text { and pig ghee } \\
\text { is placed in } \\
\text { the split. } \\
\text { Given orally } \\
\text { in mornings } \\
\text { for 3-5 days, } \\
\text { which ensures } \\
\text { the speedy } \\
\text { recovery from } \\
\text { pain and } \\
\text { wounds }\end{array}$ \\
\hline $\begin{array}{l}\text { Anorexi } \\
\text { a } \\
\text { (Thirutt } \\
\text { u Noi) }\end{array}$ & $\begin{array}{l}\text { Capsicum } \\
\text { annuum L. } \\
\text { Dalbergia } \\
\text { latifolia } \\
\text { Roxb. }\end{array}$ & $\begin{array}{l}\text { Solanaceae } \\
\text { Fabaceae }\end{array}$ & $\begin{array}{l}\text { Milahai } \\
\text { Eeti }\end{array}$ & $\begin{array}{l}\text { Shrub } \\
\text { Tree }\end{array}$ & $\begin{array}{l}\text { Fruit } \\
\text { Bark }\end{array}$ & $\begin{array}{l}\text { Decoct } \\
\text { ion } \\
\text { Decoct } \\
\text { ion }\end{array}$ & $\begin{array}{l}\text { Hot } \\
\text { water } \\
\text { Hot } \\
\text { water }\end{array}$ & $\begin{array}{l}\text { Plant parts are } \\
\text { pounded in } \\
\text { stone mortar. } \\
\text { Mixed with } \\
\text { Hot water and } \\
\text { Decoction is } \\
\text { administered } \\
\text { orally once in } \\
\text { a day for } 1 / 2 \text { lit } \\
\text { (1-kottum) up } \\
\text { to cure. }\end{array}$ \\
\hline $\begin{array}{l}\text { All ill } \\
\text { health } \\
\text { Problem } \\
\text { s }\end{array}$ & $\begin{array}{l}\text { Cassia fistula } \\
\mathrm{L} \\
\text { Clausena } \\
\text { anisata } \\
\text { (Willd.) } \\
\text { Hook.f. }\end{array}$ & $\begin{array}{l}\text { Caesalpinia } \\
\text { ceae } \\
\text { Rutaceae }\end{array}$ & $\begin{array}{l}\text { Konnai } \\
\text { Thappattai } \\
\text { chedi } \\
\text { Kundasa } \\
\text { maram }\end{array}$ & $\begin{array}{l}\text { Tree } \\
\text { Herb }\end{array}$ & $\begin{array}{l}\text { Bark } \\
\text { Bark }\end{array}$ & $\begin{array}{l}\text { Decoct } \\
\text { ion } \\
\text { Decoct } \\
\text { ion } \\
\text { Decoct } \\
\text { ion } \\
\text { Decoct } \\
\text { ion }\end{array}$ & $\begin{array}{l}\text { Hot } \\
\text { water } \\
\text { Hot } \\
\text { water } \\
\text { Hot } \\
\text { water } \\
\text { Hot } \\
\text { water }\end{array}$ & $\begin{array}{l}\text { All bark } \\
\text { materials are } \\
\text { grind well. } \\
\text { Leaf materials } \\
\text { are ground well } \\
\text { with Allium } \\
\begin{array}{l}\text { cepa, Piper } \\
\text { nigrum } \\
\text { separately and }\end{array}\end{array}$ \\
\hline
\end{tabular}




\begin{tabular}{|l|l|l|l|l|l|l|}
\hline $\begin{array}{l}\text { Dalbergia } \\
\text { latifolia } \text { Roxb. } \\
\text { Diospyros } \\
\text { montana } \\
\text { Roxb }\end{array}$ & Ebenaceae & Vekkana & & Bark & & $\begin{array}{l}\text { mixed with the } \\
\text { bark materials } \\
\text { decoction is } \\
\text { administered } \\
\text { orally about } \\
\text { one tumler } \\
\text { (kottum) day } \\
\text { for 3-5 days } \\
\text { every day in } \\
\text { the morning }\end{array}$ \\
\hline
\end{tabular}

Table 2: Fidelity level (FL)

\begin{tabular}{|c|c|c|c|c|}
\hline S. No & Botanical name & Ailment categories & $\begin{array}{l}\text { Citation for } \\
\text { particular disease } \\
\text { (Use-report) }\end{array}$ & $\begin{array}{c}\text { Fidelity level } \\
(\%)\end{array}$ \\
\hline 1 & Cassia auriculata & Dysentery with blood & 29 & 80.00 \\
\hline 2 & Cissus quadrangularis & Feeding problem & 24 & 66.66 \\
\hline 3 & Musa paradisiacal & Foot and mouth disease & 23 & 63.88 \\
\hline 4 & Evolvulus alsinoides & Wounds and injuries & 22 & 61.11 \\
\hline 5 & Andrographis paniculata & Snake bit & 19 & 52.77 \\
\hline 6 & Cassia fistula & All ill health problems & 18 & 50.00 \\
\hline 7 & Alibizia procera & Bone fracture & 17 & 47.22 \\
\hline 8 & Cleistanthus collinus & Wound & 16 & 44.44 \\
\hline 9 & Grewia asiatica & Delivery problems & 15 & 41.66 \\
\hline 10 & Capsicum аппиит & Type of fever & 14 & 38.88 \\
\hline 11 & Kielinia grandiflora & Mastitis & 13 & 36.11 \\
\hline 12 & Toddalia asiatica & Fever & 12 & 33.03 \\
\hline 13 & Endostemon viscosus & Cancer & 11 & 30.55 \\
\hline 14 & Drosera burmannii & Mesmerism & 10 & 27.77 \\
\hline 15 & Achyranthus aspera & Dog bite & 09 & 25.00 \\
\hline 16 & Tamarindus indica & Faint & 08 & 22.22 \\
\hline 17 & Coccinia indica & Yoke gal & 07 & 19.44 \\
\hline 18 & Decalepis hamiltonii & Lacation & 06 & 16.66 \\
\hline 19 & Eleasine coracana & Sprain & 05 & 13.88 \\
\hline 20 & Peperomia dindigulensis & Animal uni & 04 & 11.11 \\
\hline 21 & Borasuss flabellifer & $\begin{array}{l}\text { Eye wound due to stick } \\
\text { injuries }\end{array}$ & 03 & 08.33 \\
\hline
\end{tabular}


Table 3: Informant consensus factor

\begin{tabular}{|llccc|}
\hline S. No & Use categories & Number of taxa (Nt) & $\begin{array}{c}\text { Number } \\
\text { report (Nur) }\end{array}$ & Consensus factor \\
\hline 01 & Yoke gal & 2 & 07 & 0.83 \\
\hline 02 & Wounds and injuries & 2 & 15 & 0.92 \\
\hline 03 & Wound & 2 & 16 & 0.09 \\
\hline 04 & Sprain & 2 & 05 & 0.75 \\
\hline 05 & Faint & 2 & 08 & 0.85 \\
\hline 06 & Parturition & 2 & 15 & 0.92 \\
\hline 07 & Mesmerism & 2 & 10 & 0.88 \\
\hline 08 & Lactation & 2 & 06 & 0.08 \\
\hline 09 & Foot and mouth disease & 2 & 23 & 0.95 \\
\hline 10 & Feeding problem & 2 & 08 & 0.85 \\
\hline 11 & Eye wounds & 2 & 03 & 0.05 \\
\hline 12 & Dog bite & 2 & 09 & 0.87 \\
\hline 13 & Cancer & 2 & 04 & 0.66 \\
\hline 14 & Bone fracture & 2 & 14 & 0.92 \\
\hline 15 & Anorexia & 2 & 14 & 0.92 \\
\hline 16 & Animal uniformity & 2 & 04 & 0.06 \\
\hline 17 & Snake bite & 3 & 09 & 0.75 \\
\hline 18 & Fever & 3 & 12 & 0.81 \\
\hline 19 & Dysentery with blood & 3 & 18 & 0.89 \\
\hline 20 & Mastitis & 4 & 08 & 0.57 \\
\hline 21 & All ill health Problems & 4 & 17 & 0.81 \\
\hline
\end{tabular}

\subsection{Plants families and percentage of plats at study area}

Plants used for EVM come under 31 families. Among them Asclepiadaceae, ( 7 species $)$, predominate all other families followed by Fabaceae, (6 species), Euphorbiaceae. Rutaceae (3 species), Remaining families are represented by single or two species (Fig. 3). Most of the plants are having multiple usages, i.e., used in treating more than one ailment. Curcuma longa L and Ricinus communis are added in four herbal preparations.

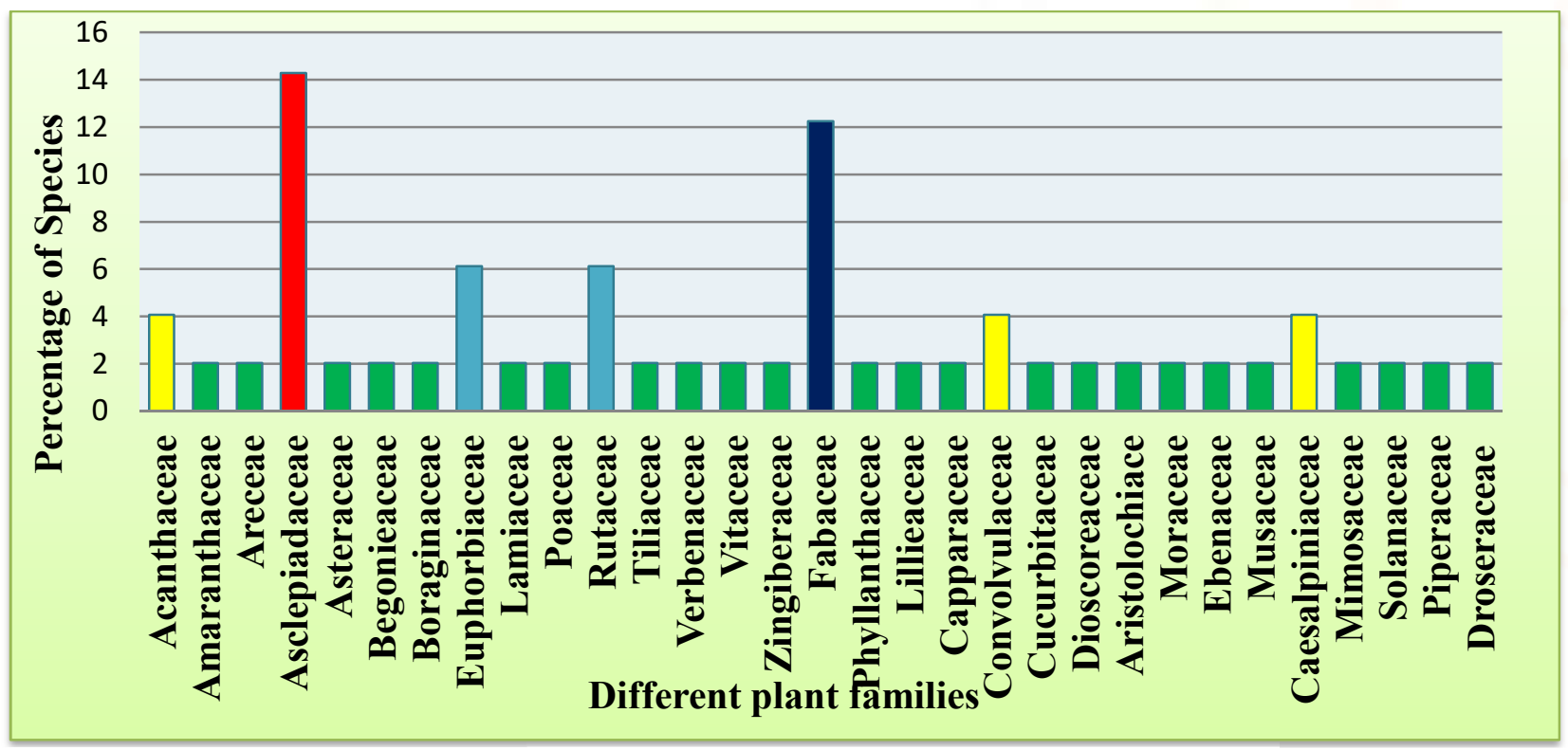

Fig 3: Representation of the families and percentage of plants at study area 


\subsection{Plant parts used}

The traditional healers of the Kalasapadi hills use various plant parts such as stem, leaf, bark, latex, oil, resin, fruit, seeds, root, rhizome, whole plant, and by-products of the plants for various medicaments. Utility value of the Leaves predominates (22) all other parts followed by Barks (7), fruits (4), root (3), stem (17), entire plant and rhizome ( 8 in each), by-products of the plants (7), latex and tender parts ( 3 in each), flower and thorn ( 2 in each), and resin (1). In 12 preparations, more than one part of the same plant is used. (Fig. 5)

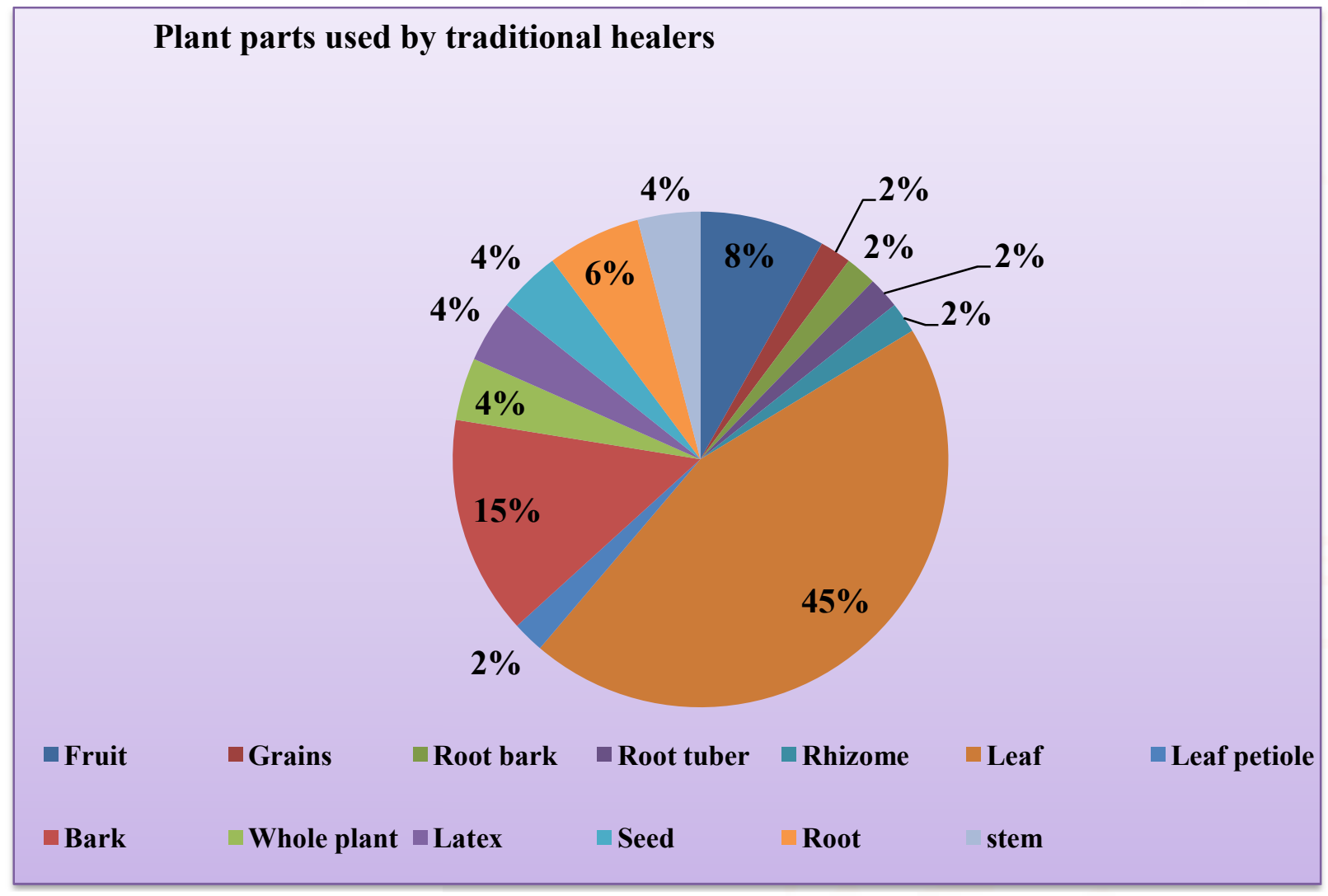

Fig 4: plants parts used by traditional healers

\subsection{Mode of preparation and administration}

As most of the traditional healers are illiterate, the knowledge on the mode of preparation and dosage of the drugs are learnt only through the long experience of the healer. For the EV health-care treatments, the native people use different methods of herbal preparations and administrations. Paste, juice, fresh parts as such and decoction are the methods commonly practiced in the study area for the preparation of EVM. In addition, some preparations include smoke or vapor forms through fumigation, processing of materials by boiling, using plant oils or soaking in water (Fig. 5). In some cases, more than one mode of preparations is also carried out.

Mode of administration also varies depends on the disease and materials used. In general, the medicines are administrated by following three types, viz., oral, external, and nasal. Oral administration is predominately used in preparations followed by external applications (52 preparations), which include administration in eyes, ears, othadam (fomentation), and fumigation process. For the control of ectoparasites, cattle sheds are fumigated regularly with dried and fresh foliages to reduce the insect pests such as ticks, mites, flies, and mosquitoes. In three preparations, both oral and external administrations are carried out and in two spiritual aspects herbal plants or preparations are not administered in any of the three modes. In the case of treating "Kunthu," materials are taken along with all ingredients in small quantities and pounded in Kal Ural (stone mortar). The paste material of an orange size is taken in a white cloth, and this preparation is dipped in urine of children below 10 years of age kept in a cup or tumbler. In the unavailability of urine, hot water is also used. However, urine is preferred for the effective treatment. Then, the preparation is taken up 
from the cup and extract oozing from the preparation through the cloth is administered as drops in ears, eyes, and nostrils for three times in both sides. Finally, solid material remained in the cloth is administered orally. It is done in early hours or in the evening hours only. This entire process is called as "Nasiam vetuthal," and it is continued for 2-3 days or up to cure. In some health problems, the herbal treatment is connected with spiritual aspects. For the processing of the herbal materials, they are using mud pots only and during the preparation, they never keep the utensils or medicinal plants directly in the ground. They hope that this practice may reduce the efficacy of the drug. Some of the preparations are carried out only in specific days such as Tuesdays, Sundays, or in Amavasai (no moon days) and can be used after 6 months only. For the treatment of udder inflammations, bloating, poison bites, and throat swellings, certain plants are used for the purpose of chanting sacred words to recover from ill health by holding the plant parts by the healers and this process is known as "Paadam Poduthal" (Chanting Manthra to recover from ill health). In $1 \mathrm{~L}$ of boiled juice, about $500 \mathrm{~g}$ of Eleasine coracana flour is added and mixed well while care must be taken to avoid the formation of any solid material. This preparation is boiled for 15 minutes until it becomes gel. This processed material is allowed to cool down and it is directly applied at bearable heat on the sprain area and left for 3 days without washing. It will be followed for 3 to 5 times at 3 days of interval. The same procedure can be followed to cure rheumatic swellings in human beings also. For the treatment of the same disease, Artocarpus hirsutus bark powder is also used instead of E. coracana in another preparation. Apart from the various ill health problems, they are protecting their mobile wealth through some general health management practices such as post-natal care of animals, protection from ecto- and endo-parasitic infections, laziness in feeding, low lactation problems, use of antiseptics, and care for insect bites. For the post-natal care, they treat animals consecutively from 2 to 21 days after delivery. The details of plants used for the treatment of various EV ailments, their preparations, administration, and dosages are given in (Table 2).

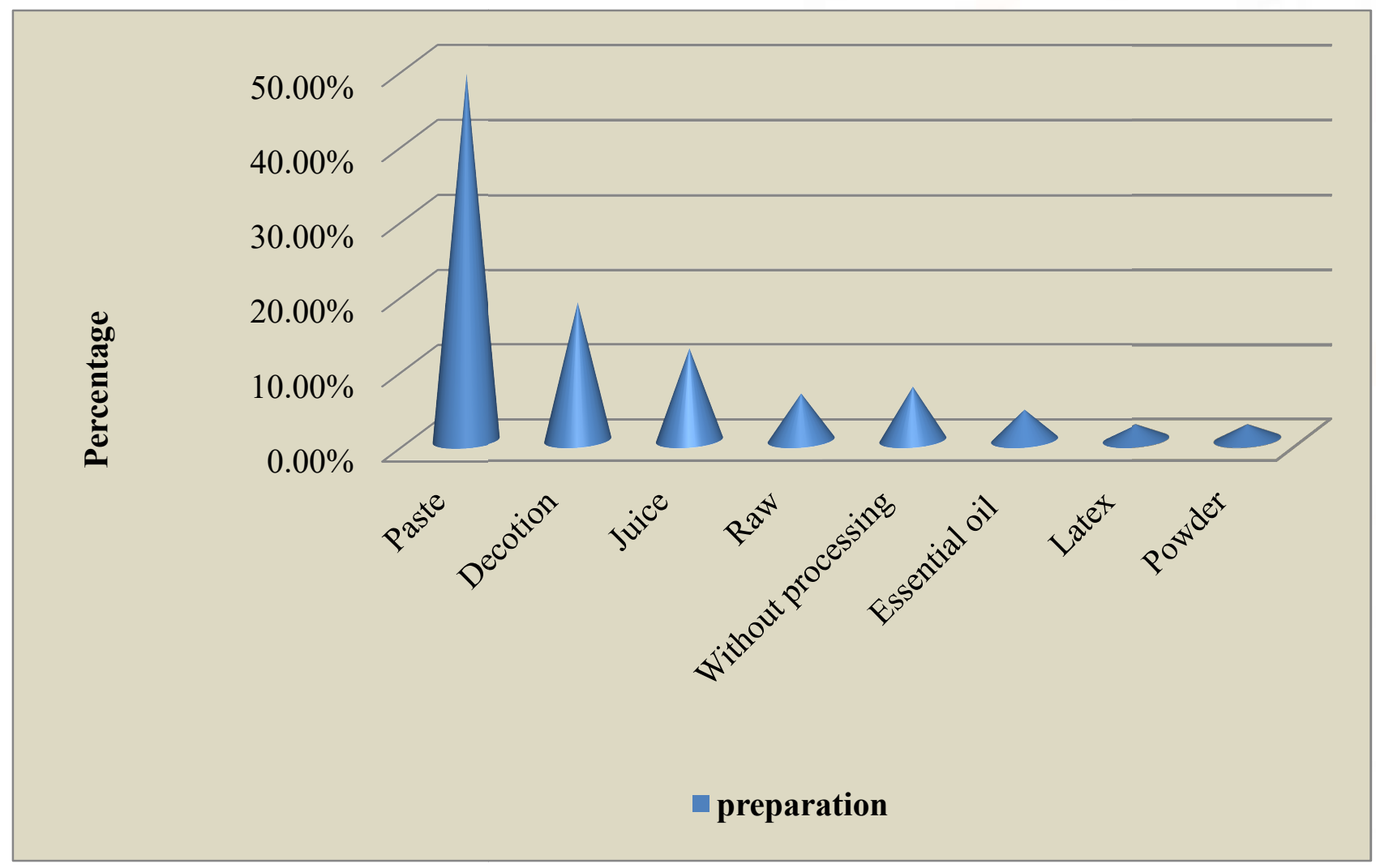

Fig. 5: Mode of preparation and administration 


\subsection{Preservatives}

Due to the seasonal availability of certain plants, there are some limitations in the practice of EVM. For which, traditional healers are preserving the preparations using certain preservatives, or they follow some procedures to preserve them for offseason uses. For the preservation of their preparations, the healers are using six plant species as ingredients, namely, Allium sativum, Cuminum cyminum, Papaver somniferum and Piper nigrum. These plants are used as ingredients, both for their medicinal values and also for their preservative properties. These plants can be gathered from wild or collected from cultivation. Preparation of herbal drugs in no moon days or in fresh mud pots are notable preservative procedures. Some of the preparations include "Saaru sakkarai" (cooking salt) as one of the ingredients for its preservative property, and the healer will not say the common word "Uppu" for cooking salt. It is concerned with the spiritual aspects.

\section{DISCUSSION}

Livestock increasing is the root of the traditional culture in the Kalasapai hills since centuries. The combination of various parts of more than 24 plants along with some preservatives is used for a single preparation in Kalasapadi hills for the treatment of more than one disease, particularly for the treatment of health infection related with "Kunthu." They hope that those combined preparations will recover the efficacy of the medicine. In the current study, 49 species of ethnoveterinary importance were recorded. There was considerable indigenous plant use knowledge among the traditional medicine practitioners of the study area. The results revealed that older traditional medicine practitioners know and use more number of EVM species than younger ones. This may indicate that the indigenous knowledge is declining among the younger generation, but could be attributed to the fact that traditional healers are reluctant to share knowledge or healing practices. This finding is in line with the work done on Mapuche community of Northwestern Patagonia, Argentina, by [40]. The study also revealed that $47 \%$ of the respondents combine more than one plant in the preparation of medicines. Plant combinations may have a synergistic effect, and could result in more effective treatment of a wider range of worms and their effects. Some $(33 \%)$ of the respondents mixed plant extracts with non plant materials such as potassium permanganate, copper sulfate, Epsom salts and table salt, and blue soap. Potassium permanganate, copper sulfate and salt are reported in literature to have been used in fish aquariums to control fish worms this phenomenon is reported by Maphosa et al [41]. Parthiban et al [42] reported that, the plants such as Achyranthes aspera, Asparagus recemosus Wild, Andrographis paniculata, Calotropis gigantean, Cardiospermum halicacabum, Cissus quadrangularis, Coccinia indica, Musa paradisiacal, Ricinus communis and Tamarindus indica are used by the scheduled castes and scheduled tribes in kudavasal taluk of Thiruvarur district. Kannan et al., [27] Curcuma longa, Artocarpus hirsutus, Cassia auriculata, Toddalia asiatica , Grewia asiatica, Limonia acidissima are used by the malayali tribes of Kalrayan hills in Salem district for the treatment of different types of diseases in livestock. According to Tolossa et al., [44] plant family Lamiaceae was most frequently useful species. In the present study area threat to the species is least as leaves are the most important plant used for medicinal purposes. It was observed that the collection of whole plant as medicinal plant from the wild were not sustainable. According to local people, this type of activity is carried out by the collectors related to illegal trade of medicinal plants. Drosera burmannii, Begonia Sp, is vulnerable to this kind of activity in the study area.

\section{CONCLUSION}

The traditional culture and indigenous knowledge of tribal in Kalasapadi hills Malayali tribal communities of the study area and they are well experienced in the administration of local herbs for the treatment of veterinary ailments. These plants are widespread, low cost and easily accessible at the time of requirement. But the practice of herbal medicine is being utilized by few ,Vaidhyarse and elderly people only. It is concluded that the healthcare system of Malayali tribe of Kalasapadi hills, traditionally depends on herbal practices for their ailments. Moreover, elders and traditional healers have rich knowledge on herbal wealth. The traditional knowledge of the healers helped us in the documentation of the ethnobotanical information. Hence, the documentation of traditional knowledge will help in the field of herbal research and inventory of new therapeutic plants for a particular therapeutic effect. At the same time the comprehensive pharmacological investigations of the herbal plants will be helpful in development of new drugs for a particular condition. 


\section{ACKNOWLEDGEMENTS}

I am very much thankful to the people of research area for sharing their knowledge, particularly the traditional healers who have taken pain in maintaining this precious tradition and valuable veterinary health science.

\section{REFERENCES}

1) Udayan S, George S, Tushar V, Balachandran I. Medicinal plants used by the Malayali Tribes of Servarayan Hills, Yercaud, Salem District, Tamil Nadu, India. Zoo's Print J. 2006; 21:2223-2224.

2) Parthipan $M$, Aravindhan V, Rajendran A. Medico-botanical study of Yercaud hills in the Eastern Ghats of Tamil Nadu, India. Anc Sci Life. 2011; 30:104-109.

3) Raul P, Pedrazo M, Manuela P. Animal health care in India. Information Centre for Low External Input in sustainable Agriculture (ILEIA). Newsletter 1990; 8(3):22-23.

4) Lalit Tiwari, C Pande. Ethnoveterinary medicines in Indian perspective: Reference to Uttarakhand, Himalaya, Indian Journal of Traditional Knowledge 2010; 9(3):611-617.

5) Dwivedi, S.K. Ethnoveterinary medicine in ancient india.Pp.103-106 in Veterinary Scince and Animal Husbandry in Ancient India .Edited by R. Somvanshi \& M.P.Yadav. IVRI, lzatnagar, india 2003.

6) Kumar, A.T. Veterinary Ayurveda in ancient indian literature Pp.8-16 in Veterinary Science and Animal Husbandry in Ancient India. Edited by R. Somvanshi \& M.P.Yadav. IVRI, lzatnagar, India 2003.

7) Mazars G, Veterinary medicines in ancient and medieval India, Studies His Med Sci. 1998; 16(12):27-36.

8) Bartha S, Quave L, Balogh L, Papp N. Ethnoveterinary practices of Covasna County, Transylvania, Romania. Journal of Ethnobiology and Ethnomedicine 2015; 11:35.

9) Sharma R, Manhas K, Magotra R. Ethno veterinary remedies of diseases among milk yielding animals in Kathua, Jammu and Kashmir, India. J. Ethnopharmacol. 2012; 141(1):265-272.

10) Bhatia H, Sharma $P$, Manhas K, Kumar K. Ethnomedicinal plants used by the villagers of district Udhampur, J\&K, India. J.Ethnopharmacol. 2014; 151(2):1005-1018.

11) Van der Merwe, D., Swan, G.E., Botha, C.J. Use of ethnoveterinary medicinal plants in cattle by
Setswana-speaking people in the Madikwe area of the North West Province of South africa. J. South African Vet. Assoc. 2001; 72 (4): 189.

12) Martin M., Mathias E., McCorkle C.M. Ethnoveterinary medicine: an annotated bibliography of community animal healthcare. ITDG Q4 publishing 2001.

13) Moyo, S., Swanepoel, F.J.C. Multifunctionality of livestock in developing communities. Role livest. Dev. Communities: Enhancing Multifunctionality 2010.

14) Abbasi, A.M., Khan, S.M., Ahmad, M., Khan, M.A., Quave, C.L., Pieroni, A et al. Botanical ethnoveterinary therapies in three districts of the Lesser Himalayas of Pakistan. J. Ethnobiol. Ethnomed. 2013; 9 (1):84.

15) Pica-Ciamarra, U., Baker, D., Morgan, N., Zezza, A. Meas. contribution Livest. Househ. Livelihoods: A Livest. Module multi-topic Househ. Survey 2011.

16) Zschocke, S., Rabe, T., Taylor, J.L., Jäger, A.K., Van Staden, J. Plant part substitution-a way to conserve endangered medicinal plants. J. Ethnopharmacol. 2000; 71:281-292.

17) Masika, P.J., Van Averbeeke, W., Sonandi, A. Use of herbal remedies by smallscale farmers to treat livestock diseases in central Eastern Cape Province, SouthAfrica. J. S. Afr. Vet. Assoc. 2000; 71:87-91.

18) Tabuti, J.R., Dhillion, S.S., Lye, K.A. Ethnoveterinary medicines for cattle (Bos indicus) in Bulamogi County, Uganda: plant species and mode of use. J.Ethnopharmacol. 2003; 88:279286.

19) Manoj Y, Anupama Y, Ekta G. Ethno veterinary practices in Rajasthan, India - A review. Int Res J Biol Sci 2012; 1(6):80-2.

20) Ashok PS, Sonawane BN, Reddy PG. Traditional ethno veterinary practices in Karanji Ghat areas of Pathradi Tahasil in Ahmednagar District, (M.S), India. Int J Plant Anim Environ Sci 2012; 1(2):649.

21) Rupeshkumar M, Kavitha K, Haldar PK. Role of herbal plants in the diabetes mellitus therapy: An overview. Int J Appl Pharm 2014; 6(3):1-3.

22) Mishra D, Patro L. Ethno veterinary practices among the rural people of Ganjam District, (Orissa), India - A case study on some common ailments. Bioscan 2010; 3:739-46.

23) Lakshminarayana V, Rao GM. Ethnoveterinary practices in north coastal districts of Andhra 
Pradesh, India. J Nat Remedies 2013; 13(2):10917.

24) Monoj Y, Ekta G. Ethnoveterinary practices by live stock owner in animal fair at Pushkar, Rajasthan, India. Int Res J Environ Sci 2014; 3(4):1-4.

25) Panda SS, Dhal NK. Plants used in ethnoveterinary medicine by native people of Nawrangpur District, Odisha, India. World J Pharm Pharm Sci 2014; 3(7):787-98.

26) Tyagai R, Sharma G, Jasuja ND, Menghani E. Indian medicinal plants as an effective antimicrobial agent. J Crit Rev 2016; 3(2):69-71.

27) Kannan M, Senthil Kumar T, Rao MV(2016) Utilization of plant resources for non-medicinal purposes by Malayali tribes of Kalrayan hills of Salem District, Tamil Nadu, IndiaInternational Journal of Herbal Medicine 2016; 4(1): 47-57.

28) Tribal barath national tribal development network2015.

29) Velayutham Saravanan. Colonial commercial forest policy and tribal private forests in Madras Presidency. Indian Economic Social History Review 2003; 40:1792-1881.

30) Santhivimalarani S, Pavadai P. Ethnoveterinary practices among the tribes of Kolli hills in Tamilnadu, India. Int J Pharm Sci Rev Res 2014; 28(2):267-71.

31) Alexiades N. Selected Guidelines for Ethnobotanical research: A Field Manual. The New York Botanical Garden, Bronx, NY 1996.

32) Martin J. Ethnobotany: A Methods Manual. Earthscan Publications Ltd., London 2004.

33) Matthew KM. The Flora of Tamil Nadu Carnatic. Vol. I-III. Tiruchirapalli, India: The Rapinat Herbarium; 1983.

34) Tan K, Wiart C. Botanical descriptions, ethnomedicinal and non-medicinal uses of the genus Artabotrys R. Br. Int J Curr Pharm Res. 2014; 6(1):34-40.

35) Heinrich M, Ankli A, Frei B, Weimann C, Sticher O: Medicinal plants in Mexico: Healers' consensus and cultural importance. Social Science and Medicine 1998; 47:1859-1871.

36) Canales M, Hernandez T, Caballero J, Romo de Vivar A, Avila G, Duran A et al. Informant consensus factor and antibacterial activity of the medicinal plants used by the people of San Rafael Coxcatlan, Puebla,Mexico. Journal of Ethnopharmacology 2005; 97:429-439.
37) Uddin Z, Hassan A. Determination of informant consensus factor of ethnomedicinal plants used in kalenga forest, Bangladesh, Bangladesh J. Plant Taxon. 2014; 21(1): 83-91.

38) Teklehaymanot $T$ : Ethnobotanical study of knowledge and medicinal plants use by the people in Dek Island in Ethiopia. Journal of Ethnopharmacology 2009; 124:69-78.

39) Bishaw M: Attitudes of modern and traditional medical practitioners toward cooperation. Ethiopian Medical Journal 1990; 28:63-72.

40) Estomba D, Ladio A, Lozada M. Medicinal wild plant knowledge and gathering patterns in Mapuche community from northwestern Patagonia. Journal of Ethnopharmacology 2006; 103:109-119.

41) Maphosa V, Masika J. Ethnoveterinary uses of medicinal plants: A survey of plants used in the ethnoveterinary control of gastro-intestinal parasites of goats in the Eastern Cape Province, South Africa, Pharmaceutical Biology, 2010; 48(6):697-702.

42) Parthiban R, Vijayakumar S, Prabhu S, Yabesh J. Quantitative traditional knowledge of medicinal plants used to treat livestock diseases from Kudavasal taluk of Thiruvarur district, Tamil Nadu, India. Revista Brasileira de Farmacognosia 2016; 26:109-121.

43) Vemban A, Subramaniam S, Gopalakrishnan K, Rao V. Major Faults/Dislocations/Lineaments of Tamil Nadu. Geol Survey of India's Misc Publication; 1977; 31:53-6.

44) Tolossa K, Debela E, Athanasiadou S, Tolera A, Ganga, Houdijk M et al. Ethno-medicinal study of plants used for treatment of human and livestock ailments by traditional in south Omo, Southern Ethiopia, Journal of Ethnobiolgy and Ethnomedicine 2013; 9:3. 